\title{
Enhancement of thermal stability of soybean oil by blending with tea seed oil
}

\author{
Nopparat Prabsangob ${ }^{1 *}$ and Soottawat Benjakul ${ }^{2}$ \\ ${ }^{1}$ Department of Product Development, Faculty of Agro-Industry, Kasetsart University, Bangkok 10900 Thailand, ${ }^{2}$ Department of Food \\ Technology, Faculty of Agro-Industry, Prince of Songkla University, Hat Yai, Songkhla 90112 Thailand
}

\section{A B S T R A C T}

\begin{abstract}
Frying accelerates oil deterioration through several chemical reactions, particularly lipid oxidation. Soybean oil (SBO), the polyunsaturated fatty acid (PUFA) rich oil, is prone to thermal degradation. Nevertheless, tea seed oil (TSO), mainly consisting of monounsaturated fatty acids (MUFA), is quite stable. This work aimed to elucidate thermal stability of SBO as affected by TSO blending at varying volume ratios. After frying for several repeated cycles, SBO/TSO blends with the ratios of 70:30, 60:40 and 50:50 showed lower total oxidative degree than SBO alone. FTIR spectra suggested less cis $\mathrm{C}=\mathrm{C}$ deformation of the SBO blended with TSO, and the 60:40 SBO/TSO blend contained the lowest secondary oxidation products. Along frying, less change in viscosity (color) was found for the 60:40 and 50:50 (60:40) SBO/TSO blends. Improved thermal stability of the blended oils was expected due to the decrease in PUFA and increase in phenolic content, and this study suggested that the 60:40 SBO/TSO blend showed the highest stability.
\end{abstract}

Keywords: Deep-frying; Oil blending; Soybean oil; Tea seed oil; Thermal stability; Oxidation

\section{INTRODUCTION}

Deep-frying is a widely operated culinary process that provides favorable characteristic to foods by maintaining flavor and juiciness within a crispy crust. Vegetable oils are generally employed as heating media in frying process. During frying, oils are exposed to elevated temperature in a presence of atmospheric oxygen and moisture from food materials, thereby accelerating oil deterioration. A series of chemical reactions are induced along frying including hydrolysis, polymerization, pyrolysis, cis-trans isomerization, double bond migration, and cyclization (Alireza et al., 2010; Romero et al., 2006). Several compounds generated adversely affect oil quality and deteriorate food properties in nutritional, safety, and sensorial aspects (Casal et al., 2010; Romero et al., 2006). Inherent characteristics of frying oils, such as fatty acid composition and contents of microconstituents, particularly tocopherols, sterols, and polyphenolics, have the profound influence on thermal stability of oils, thereby determining quality of the processed food (Casal et al., 2010).

Soybean oil (SBO), widely used as all-purpose cooking oil, possesses a good nutritive value owing to high proportion of polyunsaturated fatty acids (PUFA), particularly linoleic acid (Su and White 2004). Susceptibility of PUFA rich oils to chemical deteriorations involving oxidation and thermal degradation has been reported (Alireza et al., 2010; Casal et al., 2010; Romero et al., 2006). Improvement of oxidative stability of PUFA rich oils has been carried out through hydrogenation process that may generate trans fatty acids with health harmful effects, such as increasing total and LDL cholesterol levels (Mensink and Katan 1990). Oil blending is a promising technique to modify fatty acid profile of oils, resulting in the improved stability. Thermal tolerance of canola oil could be notably enhanced, after blending with palm oil (Alireza et al., 2010). Decrease in PUFA content of SBO could be obtained by blending with oleic rich Moringa oleifera oil, leading to significantly improved thermo-oxidative stability (Anwar et al., 2007).

Oil from tea (Camellia oleifera Abel.) seed is extensively used as cooking oil in several oriental countries. Tea seed oil (TSO) predominantly consists of monounsaturated fatty acids (MUFA), especially oleic acid (Shao et al., 2015). Higher thermo-oxidative stability of MUFA, in comparison to PUFA rich oils, has been previously reported

\footnotetext{
*Corresponding author:

Nopparat Prabsangob, Department of Product Development, Faculty of Agro-Industry, Kasetsart University, Bangkok 10900 Thailand,

E-mail: faginrpr@ku.ac.th
}

Received: 21 April 2018; $\quad$ Accepted: 28 October 2018 
(Anwar et al., 2007; Casal et al., 2010). Moreover, TSO contains several microconstituents involving polyphenols and phytosterols (e.g., stigmasterol, amyrin, and lanosterol) with several bioactivities such as antioxidant capacity, cholesterol reducibility, and anti-obesity activity (Fazel et al., 2008; Shao et al., 2015). Content of polyphenolics was positively related with thermo-oxidative resistance of cooking oils. Shao et al. (2015) reported the higher thermal stability of TSO, compared to olive oil, since TSO contained higher amounts of polyphenolics and lignins. Improvement on oxidative stability of sunflower oil could be accomplished by blending with black cumin oil containing high amount of phenolics (Kiralan et al., 2017). Moreover, by blending with the oil possessing high content of bioactive compounds, improvement on nutritive and functional value of the resulted oil could be supposed (Ramadan, 2013). Present, the restricted information about utilization of TSO to enhance thermal stability of PUFA rich oil exists. Therefore, this study aimed to elucidate the frying stability of SBO as affected by blending with TSO at different volume ratios.

\section{MATERIALS AND METHODS}

\section{Materials}

SBO (Thanakorn Vegetable Oil Products Co. Ltd., Samutprakarn, Thailand) was purchased from a local market (Songkhla, Thailand) and TSO was a product of The Chaipattana Foundation (Chiangrai, Thailand). Both oils were used without further purification. Thiobarbituric acid (TBA) and $p$-ansidine reagent were obtained from Sigma-Aldrich (St Louis, MO, USA). Trichloroacetic acid was purchased from Carlo Erba Reagenti (Rodano, Italy). Acetic acid, methanol, acetonitrile, 2-propanol, and isooctane were procured from J.T. Baker (Deventer, Netherland). All chemicals were of analytical grade, except for the reagents for HPLC analysis, which were of HPLC grade.

\section{Preparation of SBO/TSO blends and frying experiment Oil blending}

SBO was blended with TSO at various volume ratios including 80:20, 70:30, 60:40, and 50:50. The mixtures were stirred in subdued light for $1 \mathrm{~h}$. The blended oils were flushed with $\mathrm{N}_{2}$, before keeping in a plastic bottle with a screw cap at $4{ }^{\circ} \mathrm{C}$. Prior to use, the oils were stored overnight at room temperature $\left(28-30^{\circ} \mathrm{C}\right) . \mathrm{SBO}$ alone was used as the control.

\section{Frying experiment}

Frying was carried out following the method of Shi et al. (2015) with a slight modification using cotton ball as a fried material. The oil $(750 \mathrm{~mL})$ was added to a frying pod (uncovered stainless steel pan, $16 \mathrm{~cm}$ diameter, $10 \mathrm{~cm}$ height) and pre-heated at $170{ }^{\circ} \mathrm{C}$ for $10 \mathrm{~min}$. Five cotton balls $(0.45 \mathrm{~g}$ cotton each containing $1.50 \mathrm{~g}$ distilled water) were fried in the oil for $3 \mathrm{~min}$ and then removed. The absorbed oil in the cotton balls was squeezed manually. A fresh set of cotton balls was fried every $10 \mathrm{~min}$ for a period of $2.5 \mathrm{~h}$ per day. This heating program was referred to 1 frying cycle. After finishing each cycle, the oil was allowed to stand overnight, and a required amount of oil was replenished to maintain the same oil level before starting next frying cycle. The oil was heated for 3 consecutive days ( 3 repeated cycles). The fresh oil and those with $1^{\text {st }}, 2^{\text {nd }}$, and $3^{\text {rd }}$ frying cycle were cooled at room temperature for $30 \mathrm{~min}$, transferred to a screw capped bottle, flushed with $\mathrm{N}_{2}$ and closed tightly, before keeping in the dark at $-20{ }^{\circ} \mathrm{C}$. The oil samples were then subjected to analyses.

\section{Analysis of chemical composition and property of oils PUFA content}

PUFA content of the oil samples was determined using GC as per the method of Chowdhury et al. (2007). Firstly, fatty acid methyl esters (FAMEs) were prepared by adding the oil $(50 \mu \mathrm{L})$ with $\mathrm{KOH}$ solution $(0.5 \mathrm{M}$ in methanol, $1 \mathrm{~mL}$ ). After heating in a boiling water bath (Memmart, Schwabach, Germany) for $20 \mathrm{~min}$, the mixture was cooled to room temperature, before adding with a mixture of $\mathrm{HCl}$ : methanol $(4: 1 \mathrm{v} / \mathrm{v}, 0.4 \mathrm{~mL})$, deionized water $(2 \mathrm{~mL})$, and petroleum ether $(3 \mathrm{~mL})$. The distinct upper layer of the FAMEs was separated and dried with $\mathrm{N}_{2}$ flow, before re-dispersing with chloroform $(1 \mathrm{~mL})$. The sample was then introduced to GC (Agilent technologies 7890A, Wilmington, DE, USA) equipped with a flame deionized detector. Varian's capillary column (VF-5 ms, length $30 \mathrm{~m}$ $\times$ width $0.25 \mathrm{~mm} \times$ particle size $0.25 \mu \mathrm{m}$; EZ-GRIP ${ }^{\mathrm{TM}}$, Wilmington, CA, USA) was used. The column was conditioned at $180{ }^{\circ} \mathrm{C}$ for $2 \mathrm{~h}$ to attain thermal stability before use. The temperature condition was operated as following: holding at oven temperature of $150^{\circ} \mathrm{C}$ for $5 \mathrm{~min}$, increasing to $190{ }^{\circ} \mathrm{C}$ with a rate of $8{ }^{\circ} \mathrm{C} \mathrm{min}^{-1}$, increasing to $200{ }^{\circ} \mathrm{C}$ with a rate of $2{ }^{\circ} \mathrm{C} \mathrm{min}^{-1}$, and holding at $200{ }^{\circ} \mathrm{C}$ for $10 \mathrm{~min}$. Injection and detection temperatures were $250{ }^{\circ} \mathrm{C}$. Nitrogen was used as a carrier gas at a flow rate of $1 \mathrm{~mL} / \mathrm{min}$.

\section{Determination of lodine value (IV) and Acid value (AV)}

IV and AV were measured using the standard AOCS methods with analytical No. of cd 1-25 and ca 5a-40 (AOCS 1997), respectively.

Determination of total polar material (TPM) content

TPM content was evaluated according to the AOAC No. 982.27 method (AOAC 2002) following the procedure described by Schulte (2004). 


\section{Total oxidative degree (TOTOX)}

TOTOX was calculated by the following equation (De Abreu et al., 2010).

$$
\text { TOTOX }=2 P V+p-A V
$$

where $\mathrm{PV}$ is peroxide value determined using the standard AOCS method No. cd 8-53 (AOCS, 1997), and $p$-AV is para-anisidine value quantified as per the AOCS method No cd 18-90 (AOCS, 1994).

\section{Fourier transform infrared-attenuated total reflectance (FTIR-ATR) spectroscopy}

FTIR spectra of oil samples were determined by FTIRATR spectrometer (Vertex 70, Bruker, Ettlingen, Germany) using a platinum ATR diamond cell. A small aliquot of oil sample was placed onto the ATR crystal, and the spectra were recorded at $4000-400 \mathrm{~cm}^{-1}$ at 64 interferograms by using an empty ATR crystal as a background (Innawong et al., 2004).

\section{Determination of microconstituents in oils Total phenolic content (TPC)}

Firstly, phenolics were extracted from the oil samples according to the method of Parry et al. (2005) with a slight modification. The oil $(1 \mathrm{~g})$ was thoroughly mixed with a mixture of methanol: water $(9: 1,3 \mathrm{~mL})$ and centrifuged at $3000 \times \mathrm{g}$ (PrO-Research K2015, Centurion Scientific, West Sussex, UK) for $5 \mathrm{~min}$. For the same oil, the extraction was repeated for three times and the extracts were combined. The pooled extracts were dried by a rotary evaporator (Eyela N-1000, Tokyo, Japan) and stored at $-20{ }^{\circ} \mathrm{C}$ in the dark. TPC was determined by Folin Ciocalteau assay (Bali et al., 2005) and reported as milligrams gallic acid equivalent (GAE) per gram oil.

\section{Tocopherol content}

The oil $(0.15 \mathrm{~g})$ was thoroughly mixed with 2-propanol $(1 \mathrm{~mL})$. The mixture was filtered through a $0.45 \mathrm{~mm}$ pore sized filter. The filtrate $(20 \mu \mathrm{L})$ was introduced to HPLC (Agilent Technologies 1200 series G1329A, Waldbronn, Germany). Methanol and acetonitrile were used as a mobile phase at the ratio of 1:1, and the elution was performed at a flow rate of $1 \mathrm{~mL} / \mathrm{min}$. The analytical C18 column (Hypersil ODS, $5 \mathrm{~mm}$ spherical particles, $4.0 \mathrm{~mm}$ i.d. and $250 \mathrm{~mm}$ length; Agilent, Santa Clara, CA, USA) and FLD detector were used at the excitation and emission wavelengths of 295 and $325 \mathrm{~nm}$, respectively (Gliszczyńska-Świgło and Sikorska 2004). Total content of tocopherols was reported as $\mathrm{mg} \alpha$-tocopherol equivalent per gram oil.

\section{Analysis of physical property Color}

Color of the oil samples was evaluated using a HunterLab colorimeter (ColorFlex, Hunter Associates Laboratory Inc.,
VA, USA). Lightness $\left(L^{*}\right)$, redness $\left(a^{*}\right)$, and yellowness $\left(b^{*}\right)$ were measured to determine total difference in color $(\Delta E)$ as follows:

$$
\Delta E=\sqrt{\left(L_{c}^{*}-L_{0}^{*}\right)^{2}+\left(a^{*}{ }_{c}-a^{*}{ }_{0}\right)^{2}+\left(b^{*}{ }_{c}-b^{*}{ }_{0}\right)^{2}}
$$

where $L^{*}{ }_{c}, a^{*}$, and $b^{*}{ }_{c}$ are the parameters of the oil sample with different frying cycles, and $L^{*}{ }_{0}, a^{*}{ }_{0}$, and $b^{*}{ }_{0}$ are the parameters of the fresh corresponding oil.

\section{Viscosity}

Viscosity was monitored using a stress controlled rheometer (RS 75 RheoStress, Haake, Karlsruhe, Germany). A concentric cylinder system was submerged in the oil, and the force required to overcome a resistance of viscosity to rotation was measured. Shear rate was applied at $100 \mathrm{sec}^{-1}$. The measurement was performed at $25 \pm 1{ }^{\circ} \mathrm{C}$ (Sánchez-Gimeno et al., 2008). Viscosity of the oil was then determined on the basis of the speed and geometry of the probe, and relative change in viscosity at varying frying cycles, compared to the fresh oil, was reported.

\section{Statistical analysis}

All experiments were run in triplicate, and mean values with standard deviations were reported. Statistical analysis

Table 1: Fatty acid compositions and IV of SBO and SBO/TSO blends at different volume ratios as affected by various frying

\begin{tabular}{|c|c|c|c|c|}
\hline $\begin{array}{l}\text { SBO:TSO } \\
\text { ratios (v/v) }\end{array}$ & $\begin{array}{l}\text { Number of } \\
\text { frying cycle }\end{array}$ & PUFA content & IV & TPM (\%) \\
\hline TSO & 0 & $19.39 \pm 1.22^{\mathrm{D}}$ & $87.5 \pm 0.7^{\mathrm{F}}$ & $4.34 \pm 0.17^{\mathrm{C}}$ \\
\hline \multirow{4}{*}{$\begin{array}{c}\text { 100:0 } \\
\text { (control) }\end{array}$} & 0 & $60.06 \pm 0.98^{\mathrm{Aa}}$ & $122.4 \pm 0.1^{\mathrm{Aa}}$ & $8.14 \pm 0.27^{\mathrm{Ac}}$ \\
\hline & 1 & $53.59 \pm 2.01^{\mathrm{Ab}}$ & $92.9 \pm 0.2^{\mathrm{Ab}}$ & $10.21 \pm 1.13^{\mathrm{Ab}}$ \\
\hline & 2 & $41.83 \pm 2.14^{\mathrm{Ac}}$ & $73.0 \pm 0.1^{\mathrm{Ac}}$ & $12.65 \pm 0.58^{\mathrm{Aab}}$ \\
\hline & 3 & $37.47 \pm 1.98^{\mathrm{Acd}}$ & $74.9 \pm 0.1^{\mathrm{Ac}}$ & $14.12 \pm 0.94^{\mathrm{Aa}}$ \\
\hline \multirow{4}{*}{$80: 20$} & 0 & $42.65 \pm 2.00^{\mathrm{Ba}}$ & $118.8 \pm 1.1^{\mathrm{Ba}}$ & $7.67 \pm 0.29^{\mathrm{ABc}}$ \\
\hline & 1 & $37.09 \pm 1.69^{\mathrm{Bab}}$ & $77.7 \pm 0.7^{\mathrm{Bb}}$ & $7.21 \pm 0.18^{\mathrm{ABC}}$ \\
\hline & 2 & $31.97 \pm 0.97^{\mathrm{Bc}}$ & $77.5 \pm 0.6^{\mathrm{Bb}}$ & $10.95 \pm 0.35^{\mathrm{ABab}}$ \\
\hline & 3 & $27.13 \pm 2.03^{\mathrm{Bcd}}$ & $69.0 \pm 0.1^{\mathrm{Bc}}$ & $12.86 \pm 0.02^{\mathrm{ABa}}$ \\
\hline \multirow{4}{*}{ 70:30 } & 0 & $34.65 \pm 3.01^{\mathrm{BCa}}$ & $113.4 \pm 0.8^{\mathrm{Ca}}$ & $7.47 \pm 0.16^{\mathrm{ABc}}$ \\
\hline & 1 & $28.13 \pm 2.65^{\text {BCab }}$ & $68.8 \pm 1.8^{\mathrm{cb}}$ & $7.50 \pm 0.25^{\mathrm{ABc}}$ \\
\hline & 2 & $24.45 \pm 0.66^{\mathrm{BCc}}$ & $61.8 \pm 0.2^{\mathrm{ce}}$ & $9.11 \pm 0.15^{\mathrm{ABb}}$ \\
\hline & 3 & $21.67 \pm 0.21^{\mathrm{BCd}}$ & $59.7 \pm 1.2^{\mathrm{Cd}}$ & $11.98 \pm 0.06^{\mathrm{ABa}}$ \\
\hline \multirow{4}{*}{ 60:40 } & 0 & $26.25 \pm 2.21^{\mathrm{Ca}}$ & $109.1 \pm 0.1^{\mathrm{Da}}$ & $7.52 \pm 0.27^{\mathrm{Bb}}$ \\
\hline & 1 & $22.98 \pm 1.22^{\mathrm{Cb}}$ & $73.8 \pm 0.4^{\mathrm{Db}}$ & $7.55 \pm 0.39^{\mathrm{Bb}}$ \\
\hline & 2 & $19.05 \pm 0.61^{\mathrm{cbc}}$ & $69.9 \pm 3.2^{\mathrm{Dbc}}$ & $8.99 \pm 0.10^{\mathrm{Bab}}$ \\
\hline & 3 & $17.85 \pm 1.31^{\mathrm{cbc}}$ & $67.6 \pm 2.4^{\mathrm{Dc}}$ & $10.37 \pm 0.21^{\mathrm{Ba}}$ \\
\hline \multirow{4}{*}{ 50:50 } & o & $18.65 \pm 0.11^{\mathrm{CDa}}$ & $101.5 \pm 0.6^{\mathrm{Ea}}$ & $7.38 \pm 0.15^{\mathrm{Bc}}$ \\
\hline & 1 & $16.30 \pm 2.01^{\mathrm{CDa}}$ & $58.9 \pm 0.7^{\mathrm{Eb}}$ & $7.65 \pm 0.39^{\mathrm{Bc}}$ \\
\hline & 2 & $13.56 \pm 0.13^{\mathrm{CDb}}$ & $58.4 \pm 3.1^{\mathrm{Eb}}$ & $10.20 \pm 0.24^{\mathrm{Bab}}$ \\
\hline & 3 & $12.96 \pm 0.63^{\mathrm{CDb}}$ & $50.1 \pm 1.8^{\mathrm{Ec}}$ & $11.79 \pm 0.57^{\mathrm{Ba}}$ \\
\hline
\end{tabular}
cycles

For IV and TPM measurements, means $(n=3) \pm$ standard deviations are reported. The different small and capital superscripts in the same row indicate significant differences between the means within the same SBO/ TSO ratio and frying cycle $(p \leq 0.05)$, respectively. 
was performed by analysis of variance (ANOVA) using the Duncan's multiple range tests (SPSS 10.0 for windows, SPSS Inc., Chicago, IL) at a $95 \%$ confidence level.

\section{RESULTS AND DISCUSSION}

\section{Chemical characteristics of SBO/TSO blends}

PUFA content, IV and TPM of the oil samples after frying at different cycles are shown in Table 1. SBO predominantly consisted of C18:2 and C18:1, whereas TSO was mainly possessed C18:1 (data not shown). When TSO was blended with SBO, PUFA content was obviously decreased. This tendency was in agreement with the decrease in IV of SBO after blending with TSO. IV of the oil samples decreased continuously with increasing proportion of TSO in blended oils $(p \leq 0.05)$. The present result suggested that fatty acid composition of the SBO was modified by blending with TSO.

After frying, fatty acid profiles of the oil samples underwent changes indicated by decreased PUFA content, regardless of $\mathrm{SBO} / \mathrm{TSO}$ ratios. IV was also diminished with increasing numbers of frying cycles, irrespective of SBO/TSO ratios. Reduction in unstauration degree of the oils during frying generally occurred, mainly associated with destruction of $\mathrm{C}=\mathrm{C}$ through oxidation and polymerization reactions (Abdulkarim et al., 2007). This result was in accordance with other studies (Anwar et al., 2007; Naz et al., 2004). By blending with TSO, however, destruction of PUFA could be lowered suggesting to the improved thermal stability of SBO after blending with TSO. After frying for 3 repeated cycles, the relative percentages of PUFA destruction compared to the corresponding fresh oil (0 frying cycle) were $37.6,36.4,37.5,32.0$ and 30.5 for the control, 80:20, 70:30, 60:40 and 50:50 SBO: TSO blends, respectively.

As a result of frying, TPM of the oil samples increased, regardless of SBO/TSO ratios. Accumulation of polar matters such as alcohol and carbonyl compounds occurred along frying, thereby deteriorating oil quality (Romero et al., 2006; Casal et al., 2010). However, blending SBO with TSO at the ratios of 60:40 and 50:50 led to lower TPM

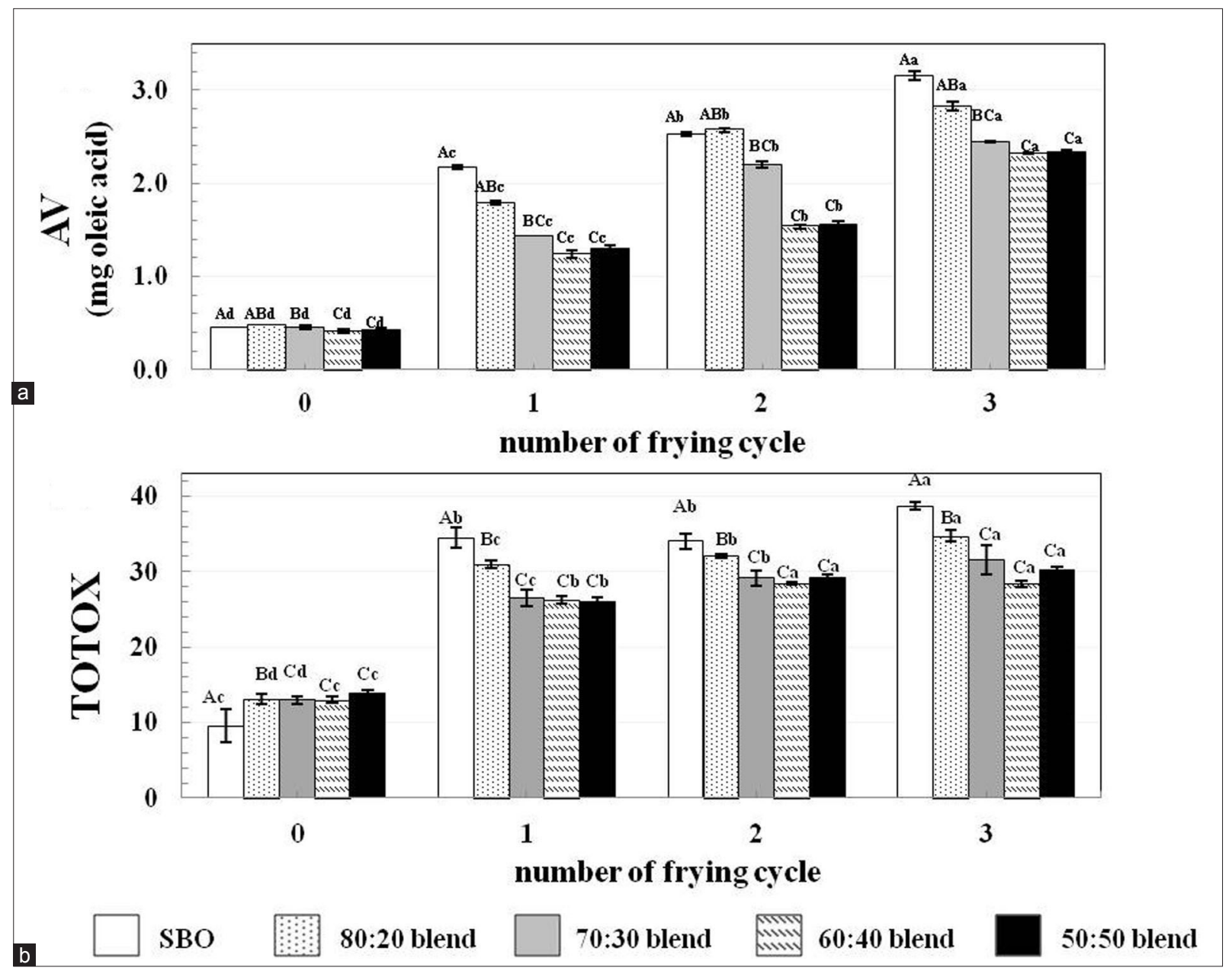

Fig 1. AV (a) and TOTOX (b) of SBO and SBO/TSO blends at different volume ratios as affected by various frying cycles. In each subfigure, means $(n=3)$ with standard deviations are reported. The different small and capital letters indicate significant differences between the means within the same SBO/TSO ratio and frying cycle $(p \leq 0.05)$, respectively. 
compared to the control $(p \leq 0.05)$, suggesting improved thermal stability of the oil samples. During frying of $25 \mathrm{~h}$, accumulation of polar matters in SBO could be diminished by increasing oleic acid content of the oil, resulting in the improved oxidative stability of the fried potato chips (Warner and Gupta, 2005).

\section{Hydrolytic and oxidation reactions in SBO/TSO blends} Effects of frying with different cycles on chemical stability indices of the blended oils are depicted in Fig. 1. Several parameters are required to monitor a series of chemical reactions occurring during frying process (Naz et al., 2004). $\mathrm{AV}$ was estimated to indicate hydrolytic degree of the oil samples (see Fig. 1a). With increasing frying cycles, AV of the oil samples continuously increased $(p \leq 0.05)$, regardless of SBO/TSO ratios. Upon exposure to high temperature in the presence of air and moisture, hydrolysis of oil is accelerated, resulting in the release of free fatty acids that are susceptible to thermal oxidation (Horuz and Maskan, 2015). AV was lowered, when SBO was blended with TSO at the ratios of $60: 40$ and 50:50 ( $p \leq 0.05)$, suggesting the improved stability against hydrolysis of the oil samples (Naz et al., 2004).

To estimate degree of thermo-oxidation, TOTOX was evaluated (see Fig. 1b). TOTOX was markedly decreased in SBO/TSO blends with the ratios of 70:30, 60:40, and
50:50 ( $p \leq 0.05)$, suggesting the improved stability of the oils. This behavior might be related with the lower PUFA content of SBO blended with TSO. Su and White (2004) reported that the $\mathrm{SBO}$ with lower $\mathrm{C} 18: 2$ content possessed higher thermal stability than that having higher content of C18:1. At the end of frying for 5 consecutive days, $p$-AV of SBO and canola oil was significantly lowered by increasing MUFA content of the oils via blending with Moringa oleifera seed oil (Abdulkarim et al., 2007). Furthermore, better stability against thermal oxidation of linoleic rich-sunflower oil could be achieved by blending with the MUFA-rich oils including clove, coriander and cumin oils (Ramadan, 2013).

\section{FTIR spectra of SBO/TSO blends}

FTIR-ATR in mid-infrared region can be promisingly used to evaluate the quality of frying oils by determining the relevant functional groups of the samples (Guillén and Cabo, 2002; Innawong et al., 2004). FTIR spectra of the selected oils are depicted in Fig. 2. The bands with strong intensity at $2929 \mathrm{~cm}^{-1}$ (asymmetric stretching $\left(\mathrm{CH}_{2}\right)$ ), $2856 \mathrm{~cm}^{-1}$ (symmetric stretching $\left.\left(\mathrm{CH}_{2}\right)\right), 1749 \mathrm{~cm}^{-1}(\mathrm{C}=\mathrm{O}$ (ester) stretching), $1464 \mathrm{~cm}^{-1}$ (C-H bending (scissoring)), and $1165 \mathrm{~cm}^{-1}$ (C-O, $\mathrm{CH}_{2}$ stretching, bending) (Innawong et al., 2004) were observed for all samples (Fig. 2a). After frying, the FTIR spectra of oils had the changes in amplitude and wave number. Guillén and Cabo (2002)
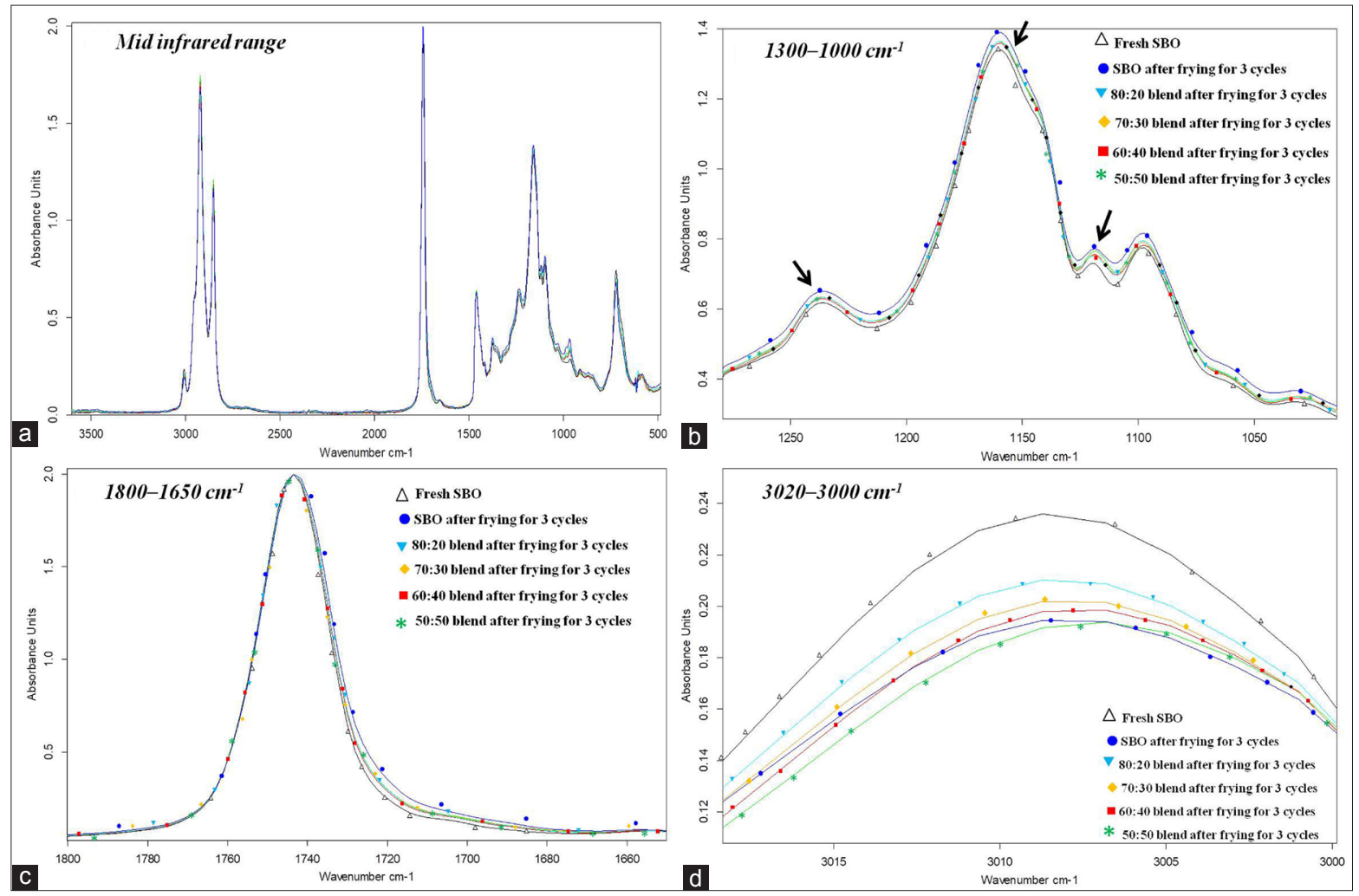

Fig 2. FTIR spectra of the selected oil samples in mid infrared range (a), wave numbers of $1300-1000 \mathrm{~cm}^{-1}(\mathrm{~b})$, wave numbers of $1800-1650$ $\mathrm{cm}^{-1}(\mathrm{c})$, and wave numbers of $3020-3000 \mathrm{~cm}^{-1}(\mathrm{~d})$. 
suggested that inferior quality of thermo-oxidized oils could be observed by the changes in FTIR spectra at the wave numbers of 1238,1163 , and $1118 \mathrm{~cm}^{-1}$. The largest change in FTIR spectra, compared to the fresh SBO, was found in SBO after frying for 3 cycles (Fig. 2b). After frying, broadening of the peak at $1749 \mathrm{~cm}^{-1}$ corresponding to the ester bond stretching was obviously noted (see Fig. 2c). Secondary oxidation products such as aldehydes and ketones showed the FTIR bands near $1728 \mathrm{~cm}^{-1}$ and might overlap with the spectra of the ester group, resulting in broadening of the peak at $1749 \mathrm{~cm}^{-1}$ (Guillén and Cabo, 2002). Relative increase in the amplitude at wave number of $1728 \mathrm{~cm}^{-1}$ of oils after frying, compared to the fresh oil, was highest for SBO (29.0\%), followed by $\mathrm{SBO} / \mathrm{TSO}$ blends with the ratios of 80:20 (14.1\%), 50:50 (13.9\%), 70:30 (13.5\%), and 60:40 (10.9\%) oils, respectively. The result suggested the lowest degree of thermo-oxidation in the 60:40 SBO/TSO blend. This trend was coincidental with TOTOX, in which the lowest value was found when $\mathrm{SBO}$ was blended with TSO at the ratios 70:30, 60:40 and 50:50.

Thermo-oxidation caused a transformation and/or disruption of cis $\mathrm{C}=\mathrm{C}$ through isomerization of cis to trans counterparts and the formation of hydroperoxides (Alireza et al., 2010; Romero et al., 2010). Decrease in the amplitude of the FTIR spectrum near $3006 \mathrm{~cm}^{-1}$ was related to a destruction of cis $\mathrm{C}=\mathrm{C}$ of oil samples (Guillén and Cabo, 2002). The FTIR spectra near $3006 \mathrm{~cm}^{-1}$ of SBO/TSO blends were illustrated in Fig. 2 d. TSO was able to lower the decrease in the amplitude of the oils after frying process. Relative decreases in the amplitude of SBO and the 80:20, 70:30, 60:40, and 50:50 blended oils after frying, compared to the fresh oil, were $15.9 \%, 9.7 \%, 12.7 \%, 13.8 \%$, and $15.7 \%$, respectively. Therefore, TSO could prevent the damage of cis $\mathrm{C}=\mathrm{C}$ of $\mathrm{SBO}$ induced by frying process.

\section{Phenolic and tocopherol contents in SBO/TSO blends}

Table 2 reveals content of tocopherols and TPC of $\mathrm{SBO}$, TSO and SBO/TSO blends at different frying cycles. The lower tocopherol content was found for TSO than that present in SBO, which was in agreement with other work (Shi et al., 2015). TSO blending caused a decrease of tocopherol content of SBO $(p \leq 0.05)$ due to the dilution effect. A positive relationship between amounts of linoleic acid and $\alpha$-tocopherol in vegetable oils has been suggested (Kalmal-Eldin and Andersson, 1997). Decreases in tocopherols with increasing numbers of frying cycle were observed, regardless of $\mathrm{SBO} / \mathrm{TSO}$ ratios $(p \leq 0.05)$, supposed due to decomposition of tocopherols, particularly with repeated frying. Although indigenous tocopherols could potently retard oxidation of oils at ambient temperature, they were not able to protect the oxidation of oils at
Table 2: Tocopherol content and TPC of SBO and SBO/TSO blends at different volume ratios as affected by various frying cycles

\begin{tabular}{|c|c|c|c|}
\hline $\begin{array}{l}\text { SBO:TSO } \\
\text { ratios }(v / v)\end{array}$ & $\begin{array}{l}\text { Number of } \\
\text { frying cycle }\end{array}$ & $\begin{array}{c}\text { Tocopherols } \\
\text { (mg } \alpha \text {-tocopherol Equiv/kg oil) }\end{array}$ & $\begin{array}{c}\text { TPC } \\
\text { (mg GAE } / \mathbf{k g} \text { oil) }\end{array}$ \\
\hline TSO & o & $747.26 \pm 22.21^{\mathrm{E}}$ & $577.7 \pm 10.9^{\mathrm{A}}$ \\
\hline \multirow{4}{*}{$100: 0$} & 0 & $1423.2 \pm 50.61^{A a}$ & $428.9 \pm 24.5^{\mathrm{CDa}}$ \\
\hline & 1 & $970.9 \pm 22.13^{\mathrm{Ab}}$ & $407.9 \pm 23.8^{\mathrm{CDab}}$ \\
\hline & 2 & $872.5 \pm 32.19^{\mathrm{Ac}}$ & $350.5 \pm 3.8^{\mathrm{CDC}}$ \\
\hline & 3 & $695.4 \pm 1.24^{\mathrm{Ad}}$ & $232.2 \pm 13.8^{\mathrm{CDd}}$ \\
\hline \multirow{4}{*}{$80: 20$} & 0 & $1065.5 \pm 3.58^{\mathrm{Ba}}$ & $462.7 \pm 19.6^{\mathrm{Ca}}$ \\
\hline & 1 & $625.0 \pm 21.55^{\mathrm{Bb}}$ & $410.2 \pm 29.0^{\mathrm{Cb}}$ \\
\hline & 2 & $472.4 \pm 9.87^{\mathrm{Bc}}$ & $356.4 \pm 34.6^{\mathrm{Cc}}$ \\
\hline & 3 & $369.8 \pm 7.17^{\mathrm{Bd}}$ & $337.6 \pm 14.8^{\mathrm{cc}}$ \\
\hline \multirow{4}{*}{ 70:30 } & 0 & $1021.5 \pm 11.57^{\mathrm{Ca}}$ & $484.4 \pm 11.0^{\mathrm{Ca}}$ \\
\hline & 1 & $586.6 \pm 25.27^{\mathrm{cb}}$ & $414.0 \pm 21.9^{\mathrm{cb}}$ \\
\hline & 2 & $264.0 \pm 2.27^{\mathrm{Cc}}$ & $393.8 \pm 23.0^{\mathrm{cb}}$ \\
\hline & 3 & $225.6 \pm 3.57^{\mathrm{Cd}}$ & $411.8 \pm 17.0^{\mathrm{cb}}$ \\
\hline \multirow{4}{*}{ 60:40 } & 0 & $891.7 \pm 7.07^{\mathrm{Da}}$ & $525.8 \pm 16.3^{\mathrm{Ba}}$ \\
\hline & 1 & $497.2 \pm 12.26^{\mathrm{Db}}$ & $513.5 \pm 9.6^{\mathrm{Ba}}$ \\
\hline & 2 & $353.0 \pm 8.17^{\mathrm{Dc}}$ & $495.7 \pm 23.6^{\mathrm{Ba}}$ \\
\hline & 3 & $256.0 \pm 2.88^{\mathrm{Dd}}$ & $428.1 \pm 10.1^{\mathrm{Bb}}$ \\
\hline \multirow{4}{*}{$50: 50$} & $\mathbf{0}$ & $750.9 \pm 11.29^{\text {Еа }}$ & $539.9 \pm 9.6^{\mathrm{Ba}}$ \\
\hline & 1 & $471.7 \pm 21.47^{\mathrm{Eb}}$ & $529.9 \pm 18.0^{\mathrm{Bab}}$ \\
\hline & 2 & $331.3 \pm 3.67^{\mathrm{Ec}}$ & $450.7 \pm 23.9^{\mathrm{Bc}}$ \\
\hline & 3 & $240.3 \pm 1.58^{\mathrm{Ed}}$ & $436.0 \pm 33.4^{\mathrm{Bc}}$ \\
\hline
\end{tabular}

Means $(n=3) \pm$ standard deviations are reported. The different small and capital superscripts in the same column indicate significant differences between the means within the same SBO/TSO ratio and frying cycle $(p \leq 0.05)$, respectively.

elevated temperature condition (Ghafoorunissa, 2007). Disappearance of $\alpha$-tocopherol in sunflower oil was observed after 8 days of storage at $60^{\circ} \mathrm{C}$ (Kiralan et al., 2017). Decomposition of tocopherols in olive oil was observed after 3-6 h of frying (Casal et al., 2010). Considering the content of phenolic compounds, TSO possessed significantly higher TPC than SBO $(p \leq 0.05)$. The SBO/TSO blends with the ratios of 60:40 and 50:50 contained the higher TPC compared to other blends $(p \leq 0.05)$. TSO is a good source of phenolics with a potent antioxidant activity, especially epigallocatechin gallate (Shi et al., 2015). TPC was generally decreased with increasing numbers of frying cycle. However, the 60:40 SBO/TSO blend could retain TPC up to the end of second frying cycle $(p>0.05)$. This behavior was coincidental with its lowered TOTOX (see Fig. 1b) and less formation of the secondary oxidation products as suggested by the FTIR spectra (see Fig. 2c).

When the correlations between some selected parameters were examined (Fig. 3a), higher negative correlation between IV and TOTOX was noted, compared to that between IV and AV. This suggested higher susceptibility of the unsaturated oils against thermo-oxidation, compared to heat induced hydrolysis. Among a series of several chemical reactions occurring during frying, thermo-oxidation was regarded as a major reaction affecting the deterioration of oils (Farag et al., 2007). 
The relationship between IV and the residual TPC or tocopherols of the oil samples was observed (Fig. 3b). Positive correlation between IV and remaining tocopherols was found, suggesting that frying greatly lowered IV and destroyed tocopherols in oils. This result was in agreement with the report of Verleyen et al., (2002). On the other hands, the remaining TPC seemed to be independent with IV, implying the higher thermal tolerance of phenolics than tocopherols. Although tocopherols play a role as major antioxidants in vegetable oils, loss of their antioxidative capacity was reported at temperature above $100{ }^{\circ} \mathrm{C}$ (Kalmal-Eldin, 2006).

The relationship between tocopherols and AV or TOTOX, and the plot between TPC and AV or TOTOX are shown in Fig. 3c and Fig. 3d, respectively. Negative relationships were observed for these plots, suggesting the role of tocopherols and TPC in enhancement of thermal stability of the oils. This tendency was in agreement with other work (Kiralan et al., 2017). It was worth noting that higher $\mathrm{R}^{2}$ was observed for the relationship between TPC and AV or TOTOX, compared to the plot between tocopherols and AV or TOTOX. The higher efficiency of phenolics in delaying thermal degradation of the oils, compared to tocopherols was presumed. This was plausibly due to higher thermal tolerance of phenolics. Ramadan (2013) determined degree of thermo oxidation of sunflower oil as affected by blending with various MUFA-rich oils (i.e., clove, coriander, cumin, and black cumin oils). The highest stability was observed when the sunflower oil was blended with clove oil attributed due to the highest phenolic content of the clove oil compared to other selected MUFA-rich oils. By mixing with black cumin oil, oxidative stability against heating at $110^{\circ} \mathrm{C}$ of sunflower oil could be enhanced which was explained by the abundant presence of phenolic compounds in the black cumin oil, especially thymoquinone (Kiralan et al., 2017). Thermooxidative stability of olive oils could be enhanced owing to a presence of phenolic compounds (Casal et al., 2010). Higher contents of phenolics and other phytosterols, e.g., stigmasterol, amyrin, and lanoserol, etc. were reported for TSO, compared to olive oil. This was coincidental with higher thermal stability of the former: decomposition temperatures of TSO and olive oil in air atmospheric

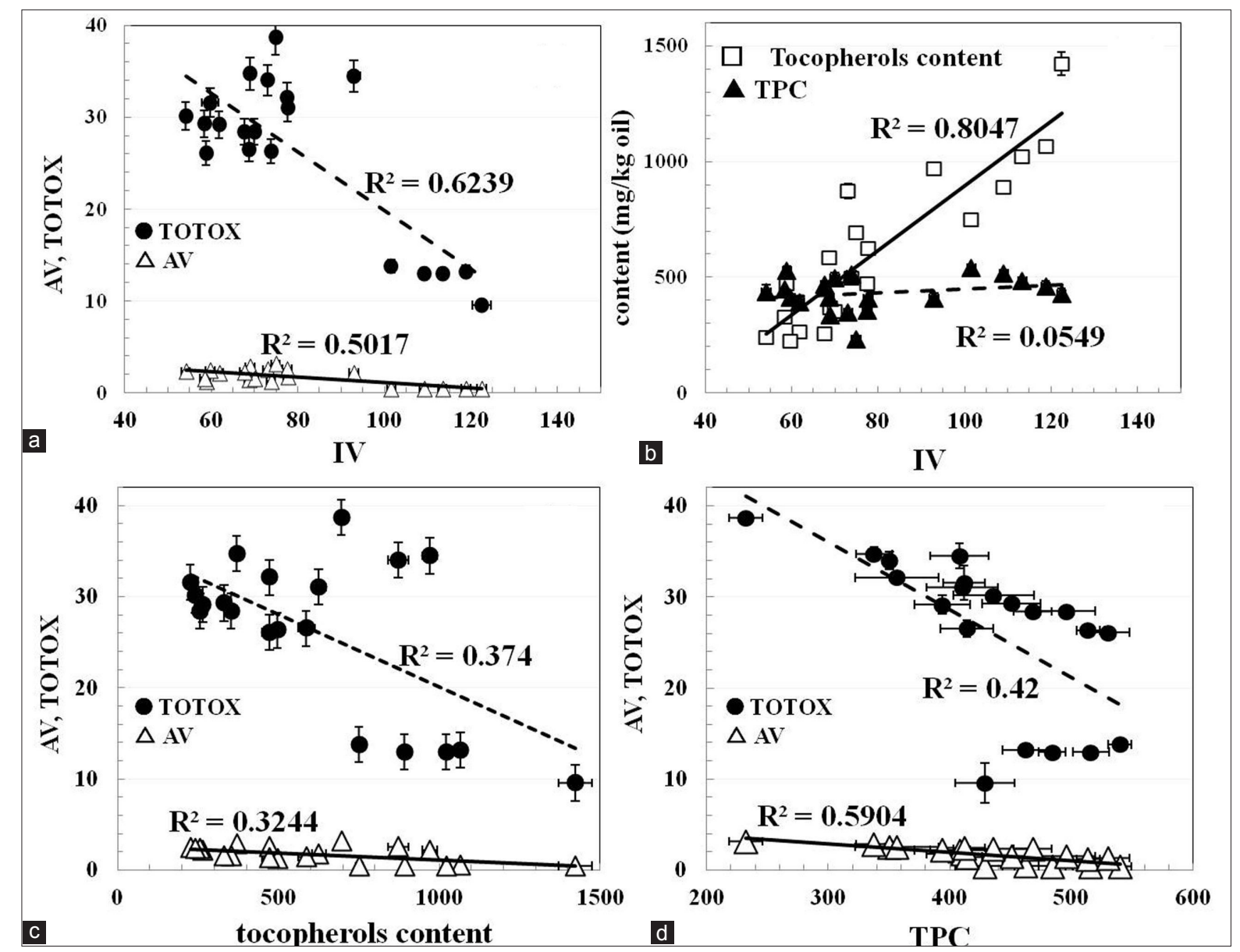

Fig 3. Correlation between IV and AV or TOTOX (a), IV and TPC or tocopherol content (b), tocopherol content and AV or TOTOX (c), and TPC and AV or TOTOX (d) of the oil samples. 


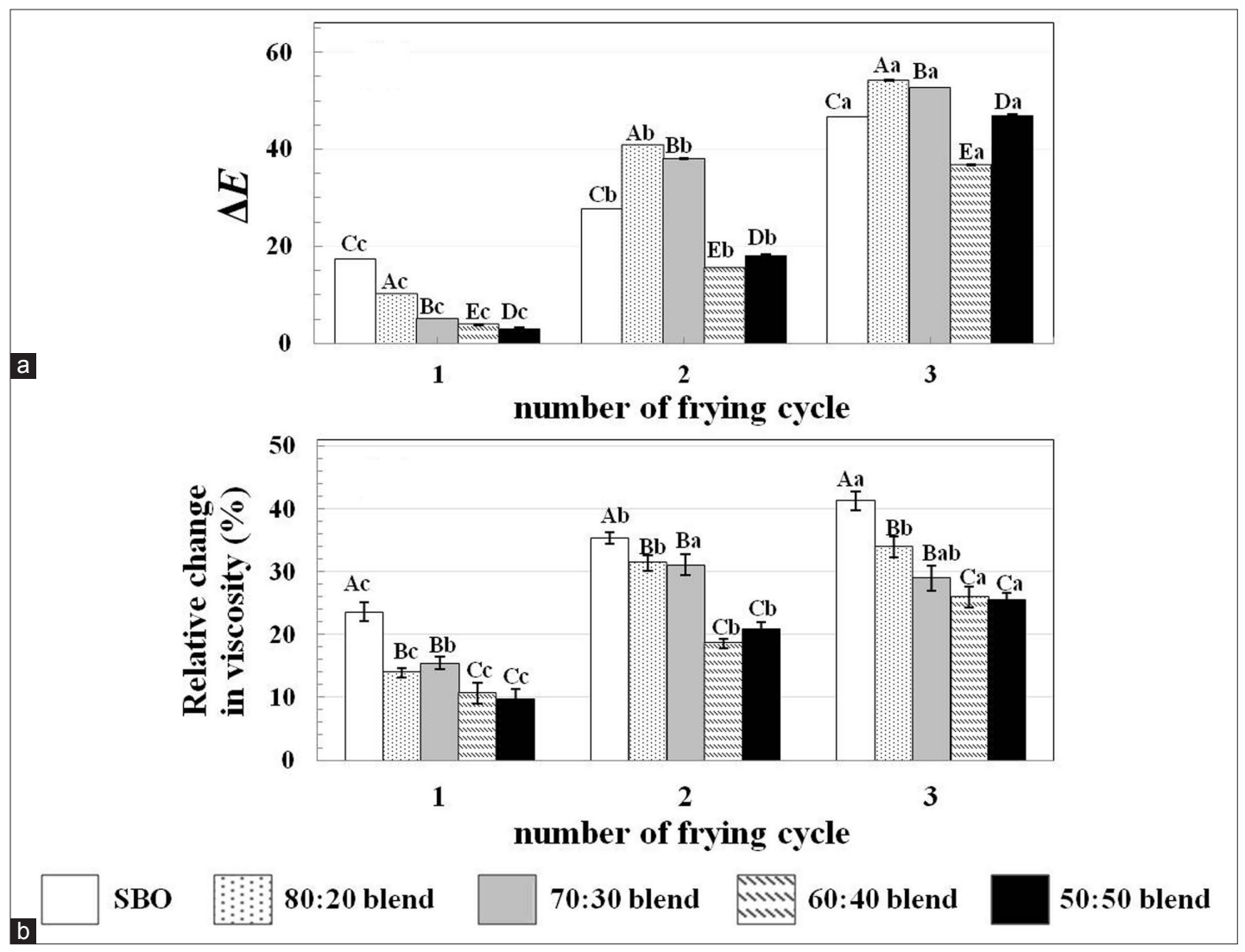

Fig 4. $\Delta E$ (a) and relative change in viscosity (b) of $\mathrm{SBO}$ and SBO/TSO blends at different volume ratios as affected by various frying cycles. In each subfigure, means $(n=3) \pm$ standard deviations are reported. The different small and capital letters indicate significant differences between the means within the same SBO/TSO and frying cycle $(p \leq 0.05)$, respectively.

condition were $374{ }^{\circ} \mathrm{C}$ and $237.5^{\circ} \mathrm{C}$, respectively (Shao et al., 2015). Improvement on thermal stability of sunflower oil could be achieved by incorporating phenolic richolive leaves extracts ( $0-2400 \mathrm{ppm})$, as indicated by the increased smoke point and lowered oxidative indices of the sunflower oil added with the extracts, especially at higher concentration (Farag et al., 2007). Due to their thermal tolerance, polyphenols could delay degradation of tocopherol during deep-frying process, thereby enhancing thermal stability of the frying oil (Pellegrini et al., 2001).

\section{Physical properties of SBO/TSO blends}

Physical changes of SBO and SBO/TSO blends as affected by frying monitored by $\Delta E$ and relative change in viscosity at varying frying cycles are shown in Fig. $4 \mathrm{a}$ and Fig. 4b, respectively. Increased color intensity of oils with increasing frying cycles could be attributed to the accumulation of non-volatile decomposition products such as oxidized triacylglycerols and free fatty acids, indicating the higher oil deterioration (Abdulkarim et al., 2007; Su and White, 2004). $\Delta E$ continuously increased with increasing numbers of frying cycle $(p \leq 0.05)$, irrespective of SBO/TSO ratios. The lowest $\Delta E$ was evident for the 60:40 SBO/TSO blend $(p \leq 0.05)$, which was in agreement with its lowest accumulated secondary oxidation products as previously illustrated by the FTIR spectra.

Relative change in viscosity was generally augmented with increasing numbers of frying cycle, regardless of $\mathrm{SBO} / \mathrm{TSO}$ ratios (see Fig. 4b). After frying, increase in oil viscosity more likely resulted from polymerization and formation of high molecular weight compounds (Abdulkarim et al., 2007; Su and White, 2004). Lowered relative change in viscosity was observed for the 60:40 and 50:50 SBO/TSO blends ( $p \leq 0.05$ ), which was in correspond with the lowered TPM of these oil samples compared to the others (see Table 1). The present results suggested the improved thermal stability of SBO by blending with TSO at the sufficient ratio. Lower degrees of polymerization and other related reactions of the oil with higher oleic acid content were reported (Su and White, 2004). After frying at $180^{\circ} \mathrm{C}$ for 5 days, higher rate of oil darkening was observed for PUFA rich oils (SBO and canola oil), compared to the MUFA rich oil (M. oleifera seed oil) (Abdulkarim et al., 2007). Lowered $\Delta E$ and viscosity increment of the $60: 40$ and 50:50 SBO/TSO blends, compared to SBO might be associated with the presence of phenolic compounds. By mixing with phenolic rich olive leaves extracts, change in 
color and viscosity along frying for 5 days of sunflower oil could be retarded (Farag et al., 2007).

Thermal stability of SBO could be enhanced by blending with TSO at sufficient amount. Phenolics and other bioactive compounds present in TSO might increase thermal stability of SBO/TSO blends. It has been reported that TSO is rich in lignans, especially sesamin and 2,5-bis-benzo[1,3] dioxol-5-yl-tetrahydro-furo[3,4-d] $[1,3]$-dioxine (Lee and Yen, 2006). Greater heat tolerance of sesamin, compared to tocopherols, has been reported. At the end of heating $\left(180^{\circ} \mathrm{C}\right.$ for $\left.120 \mathrm{~min}\right)$, retention percentage of tocopherols in SBO was increased from 25 to $40 \%$, when sesamin $(1.2 \%)$ was incorporated into the oil (Ghafoorunissa, 2007). Lignans were converted to several derivatives with effective antioxidant activity, thereby improving thermal stability of frying oils (Ghafoorunissa, 2007; Nakai et al., 2003).

\section{CONCLUSIONS}

Thermal stability SBO could be successfully improved by blending with TSO by decreasing PUFA content and increasing amount of phenolic compounds. Higher thermal tolerance of phenolics in TSO in comparison to tocopherols in SBO mainly contributed to stability of the blends. By blending with TSO, cis $\mathrm{C}=\mathrm{C}$ deformation of SBO was retarded. The effective SBO: TSO blending ratio was $60: 40$ as suggested by the lowest changes in color and viscosity, lowered TOTOX, as well as the lowest accumulated content of secondary oxidation products.

\section{ACKNOWLEDGEMENT}

This research was supported grants from The Thailand Research Fund (contract no. MRG5980106).

\section{Authors' Contributions}

$\mathrm{NC}$ (corresponding author) conducted the research work, interpreted the data, and prepared the manuscript. SB provided the guidance for experimental design and critically reviewed the manuscript.

\section{REFERENCES}

Abdulkarim, S. M., K. Long, O. M. Lai, S. K. S. Muhammad and H. M. Ghazali. 2007. Frying quality and stability of high-oleic Moringa oleifera seed oil in comparison with other vegetable oils. Food Chem. 105: 1382-1389.

Alireza, S., C. P. Tan, M. Hamed and Y. B. C. Man. 2010. Effect of frying process on fatty acid composition and iodine value of selected vegetable oils and their blends. Int. Food Res. J. 17: 295-302.

Anwar, F., A. I. Hussain, S. Iqbal and M. I. Bhanger. 2007.
Enhancement of the oxidative stability of some vegetable oils by blending with Moringa oleifera oil. Food Chem. 103: 1181-1191.

AOCS. 1994. Official Methods and Recommended Practices of the American Oil Chemists' Society Method Cd 18-90, AOCS Press, Champaign.

AOCS. 1997. Official Methods and Recommended Practices of the American Oil Chemists' Society Method Cd 1-25, Ca 5a-40, Cd 8-53, AOCS Press, Champaign.

AOAC. 2002. Polar Components in Frying Fats. Official Method 982.27. Association of Official Analytical Chemists, Arlington.

Bali, S., G. Stuebiger, S. Krist, H. Unterweger and G. Buchbauer. 2005. Characterization of various grape seed oils by volatile compounds, triacylglycerol composition, total phenols, and antioxidant capacity. Food Chem. 108: 1122-1132.

Casal, S., R. Malheiro, A. Sendas, B. P. P. Oliveira and J. A. Pereira. 2010. Olive oil stability under deep-frying conditions. Food Chem. Toxicol. 48: 2972-2979.

Chowdhury, K., L. A. Banu, S. Khan and A. Latif. 2007. Studies on the fatty acid composition of edible oil. Bangladesh J. Sci. Ind. Res. 42: 311-316.

De Abreu, D. A. P., P. P. Losada, J. Maroto and J. M. Cruz. 2010. Evaluation of the effectiveness of a new active packaging film containing natural antioxidants (from barley husks) that retard lipid damage in frozen Atlantic salmon (Salmo salar L.). Food Res. Int. 43: 1277-1282.

Farag, R. S., E. A. Mahmoud and A. M. Basuny. 2007. Use crude olive leaf juice as a natural antioxidant for the stability of sunflower oil during heating. Int. J. Food Sci. Technol. 42: 107-111.

Fazel, M., M. A. Sahari and M. Barzegar. 2008. Determination of main tea seed oil antioxidants and their effects on common kilka oil. Int. Food Res. J. 15: 209-217.

Ghafoorunissa, S. H. 2007. Sesame lignans enhance the thermal stability of edible vegetable oils. Food Chem. 105: 1076-1085.

Gliszczyńska-Świgło, A. and E. Sikorska. 2004. Simple reversedphase liquid chromatography method for determination of tocopherols in edible plant oils. J. Chromatogr. A. 1048: 195-198.

Guillén, M. D. and N. Cabo. 2002. Fourier transform infrared spectra data versus peroxide and anisidine values to determine oxidative stability of edible oils. Food Chem. 77: 503-510.

Horuz, T. I. and M. Maskan. 2015. Effect of the phytochemicals curcumin, cinnamaldehyde, thymol and carvacrol on the oxidative stability of corn and palm oils at frying temperatures. J. Food Sci. Technol. 52: 8041-8049.

Innawong, B., P. Mallikarjunan, J. Irudayaraj and J. E. Marcy. 2004. The determination of frying oil quality using fourier transform infrared attenuated total reflectance. LWT Food Sci. Technol. 37: 23-28.

Kalmal-Eldin A. 2006. Effect of fatty acids and tocopherols on the oxidative stability of vegetable oils. Eur. J. Lipid Sci. Technol. 108: 1051-1061.

Kalmal-Eldin, A. and R. Andersson. 1997. A multivariate study on the correlation between tocopherol content and fatty acid composition in vegetable oil. J. Am. Oil Chem. Soc. 74: 375-380.

Kiralan, M., M. Ulaş, A. Özaydin, N. Özdemir, G. Özkan, A. Bayrak and M. F. Ramadan. 2017. Blends of cold pressed black cumin oil and sunflower oil with improved stability: A study based on changes in the levels of volatiles, tocopherols and thymoquinone during accelerated oxidation conditions. J. Food Biochem. 41: e12272.

Lee, C. P. and G. C. Yen. 2006. Antioxidant activity and bioactive compounds of tea seed (Camellia oleifera Abel.) oil. J. Agric. Food Chem. 54: 779-784. 
Mensink, R. P. and M. B. Katan. 1990. Effects of dietary trans fatty acids on high-density and low-density lipoprotein cholesterol levels in healthy subjects. New Engl. J. Med. 323: 439-445.

Nakai, M., M. Harada, K. Nakahara, K. Akimoto, H. Shibata, W. Miki and Y. Kiso. 2003. Novel antioxidative metabolites in rat liver with ingested sesamin. J. Agric. Food Chem. 51: 1666-1670.

Naz, S., H. Sheikh, S. Siddiqi and S. A. Sayeed. 2004. Oxidative stability of olive, corn and soybean oil under different conditions. Food Chem. 88: 253-259.

Parry, J., L. Su, M. Luther, K. Zhou, M. P. Yurawecz, P. Whittaker and L. Yu. 2005. Fatty acid composition and antioxidant properties of cold-pressed marionberry, boysenberry, red raspberry, and blueberry seed oils. J. Agric. Food Chem. 53: 566-573.

Pellegrini, N., F. Visioli, S. Buratti and F. Brighenti. 2001. Direct analysis of total antioxidant activity of olive oil and studies on the influence of heating. J. Agric. Food Chem. 49: 2532-2538.

Ramadan, M. F. 2013. Healthy blends of high linoleic sunflower oil with selected cold pressed oils: Functionality, stability and antioxidative characteristics. Ind. Crops Prod. 43: 65-72.

Romero, A., S. Bastida and F. J. Sanchez-Muniz. 2006. Cyclic fatty acid monomer formation in domestic frying of frozen foods in sunflower oil and high oleic acid sunflower oil without oil replenishment. Food Chem. Toxicol. 44: 1674-1681.
Sánchez-Gimeno, A. C., A. I. Negueruela, M. Benito, A. Verce and R. Oria. 2008. Some physical changes in Bajo Aragón extra virgin olive during the frying process. Food Chem. 110: 654-658.

Schulte, E. 2004. Economic micromethod for determination on polar components in frying fats. Eur. J. Lipid Technol. 106: 772-776.

Shao, P., Q. Liu, Z. Fang and P. Sun. 2015. Chemical composition, thermal stability and antioxidant properties of tea seed oils obtained by different extraction methods: Supercritical fluid extraction yields the best oil quality. Eur. J. Lipid Sci. Technol. 117: 355-365.

Shi, R., Y. Guo, F. Vriesekoop, Q. Yuan, S. Zhao and H. Liang. 2015. Improving oxidative stability of peanut oil under microwave treatment and deep fat frying by stearic acid-surfactant-tea polyphenols complex. Eur. J. Lipid Sci. Technol. 117: 1008-1015.

Su, C. and P. White. 2004. Frying stability of high-oleate and regular soybean oil blends. J. Am. Oil Chem. Soc. 81: 783-788.

Verleyen, T., A. Kamal-Eldin, R. Mozuraityte, R. Verhe, K. Dewetinck, A. Huyghebaert and W. De Greyt. 2002. Oxidation at elevated temperatures: Competition between-tocopherol and unsaturated triacylglycerols. Eur. J. Lipid Sci. Technol. 104: 228-233.

Warner, K. and M. Gupta. 2005. Potato chip quality and frying oil stability of high oleic acid soybean oil. J. Food Sci. 70: 395-400. 